\title{
Evaluación financiera del valor generado por un sistema de información en una empresa de transportes
}

\author{
Financial evaluation of the value generated by an information \\ system in a transport firm \\ Pedro Manuel Sosa San Martín ${ }^{1} \quad$ Emigdio Antonio Alfaro Paredes ${ }^{1}$ \\ Recibido 8 de abril de 2010, aceptado 22 de noviembre de 2011 \\ Received: April 8, 2010 Accepted: November 22, 2011
}

\begin{abstract}
RESUMEN
En este estudio se evaluó el retorno financiero de la implantación de un sistema de información de venta de envío de paquetes (encomiendas) desarrollado in-house en una empresa de transporte interprovincial de carga en Lima mediante la variación del procedimiento 059 de la metodología MAIGTI, el cual sugiere evaluar el valor actual neto considerando ingresos adicionales (margen de contribución adicional y ahorros) y egresos adicionales (inversiones adicionales y gastos adicionales) con una tasa mínima atractiva de retorno en un período de evaluación similar al período de planificación de largo plazo según el tipo de entidad en que se realizó la evaluación. La implantación del sistema de información de encomiendas dio como resultado para el primer año un valor actual neto de S/. 138.070 nuevos soles (aproximadamente US\$ 48.446) y llegando para el tercer año a un valor actual neto de S/. 1.178.996 nuevos soles (aproximadamente US\$ 413.683), gracias al aumento de la lealtad del cliente debido a una reducción del tiempo al $70 \%$ en la emisión de comprobantes y evitar pérdidas debido a que una proporción de los clientes no contrataban el servicio porque la emisión de facturas era manual. El estudio demostró también que la variación del procedimiento 059 de la metodología MAIGTI es válida para la evaluación del retorno financiero de un sistema de información en una empresa de transportes.
\end{abstract}

Palabras clave: Valor, sistema de información, MAIGTI, metodología, evaluación financiera.

\section{ABSTRACT}

In this study was evaluated the financial results after the implantation of an information system for selling shipping of packages, developed in-house in an inter-carrier charge in Lima through the variation of procedure 059 of MAIGTI methodology, which suggests the evaluation of the net present value considering additional incomes (contribution margin and savings) and additional expenses (additional investments and additional expenditures) with a discount rate in a evaluation period similar to long term planning period according to the type of entity in which the evaluation was realized. The implantation of the information system of packages shipping obtained S/. 138,070 new soles (approximately US\$ 48,446) as net present value after the first year, and S/. 1,178,996 new soles (approximately US\$ 413,683) as net present value after the third year, due the increment of loyalty of clients through the reduction of time to $70 \%$ in the emission of bills and for avoiding losses due a proportion of clients didn't hire the service because the emission of bills was manual. Also, the study demonstrates that the variation of procedure 059 of the MAIGTI methodology is valid for the evaluation of financial return of an information system in a transport firm.

Keywords: Value, information system, MAIGTI, methodology, financial evaluation.

1 Escuela de Ingeniería de Sistemas e Informática. Universidad Privada Norbert Wiener. Jr. Larrabure y Unanue 110 Urb. Santa Beatriz.Lima 1, Perú. E-mail: a2003100406@uwiener.edu.pe; emigdio_alfaro@yahoo.com 


\section{INTRODUCCIÓN}

El desarrollo de un sistema de información demanda una elevada inversión; sin embargo, las organizaciones comúnmente carecen de cálculos del beneficio económico del desarrollo y la implantación de un sistema de información, justificándose en muchos casos por la complejidad para desarrollar dichos cálculos. Además, cuando se realizan los cálculos de los presupuestos de tecnologías de información, ocurrirían diversos errores, como lo señaló Huber [7], analista de la firma Gartner, quien indicó que el $20 \%$ del presupuesto en tecnología de información se realiza de forma equivocada e ineficaz, siendo la inversión mundial en tecnologías de información, aproximadamente US\$ 500 billones. Asimismo, Lucas [10] explicó el alto riesgo para muchas organizaciones como la NationsBank, que en 1997 ya gastaba US\$ 500.000.000 anuales en software y tenía un presupuesto total para tecnología de información de US\$ 1.900.000.000.

Gantin [5] indicó que la elevada inversión en tecnologías de información podría justificarse de la siguiente manera: a) reducir o evitar costos, b) aumentar o mantener los ingresos, c) crear capacidades para futuros ingresos, d) mejorar el proceso de toma de decisiones, y e) cumplir con la normativa. Una encuesta trimestral de la entidad de servicio de información "e-Skills" del Reino Unido, en una investigación sobre las tecnologías de información y comunicaciones o TIC realizada por McCue [11], demostró que sólo el $11 \%$ de las empresas mide su retorno de inversión, el 89\% restante de las empresas utiliza la "intuición" o el "adivinar".

Para las dificultades de medir el retorno de inversión el informe de situación mundial del IT Governance Institute [9] afirmó que los mayores obstáculos están relacionados con la falta de conocimientos sobre las prácticas de gobernanza en tecnología de información y los resultados desconocidos de las prácticas de gestión de inversiones en tecnología de información en términos de retorno de la inversión.

Esta investigación estuvo enfocada en un sistema de información de carga en una empresa de transporte interprovincial de Lima denominada "TransProv" (pseudónimo por razones de confidencialidad). El giro de carga ha experimentado vertiginosidad en su demanda en los últimos años. La entidad contaba con un sistema que sólo cubría los procesos de venta en Lima; en provincias, los demás procesos eran manuales existiendo problemas operativos. En septiembre de 2005 se inició un nuevo proyecto de desarrollo de sistema de información bajo sus propios recursos (In-House) y algunos años después de la implantación, "TransProv" deseaba evaluar el costo/beneficio del sistema de información, para tomar mejores decisiones futuras.

\section{REVISIÓN DE LA LITERATURA}

A continuación se describen brevemente algunas metodologías para hallar el retorno financiero:

- Hubbard [6] presentó la Metodología Applied Information Economics (AIE), desarrollada por Doug Hubbard, basada en las herramientas de la economía, la teoría financiera y las estadísticas.

- Alfaro [2] presentó MAIGTI (Metodología para la Auditoría Integral de la Gestión de la Tecnología de Información), la cual contiene un procedimiento para el cálculo de generación de valor de los proyectos denominado 059 en el cual se especifican los siguientes elementos para evaluar la conveniencia de un proyecto de inversión en Tecnología de Información: a) período a evaluar, b) tasa mínima atractiva de retorno de la inversión, c) ingresos adicionales, d) gastos adicionales, e) flujo neto, f) generación de valor del proyecto y g) verificar la conveniencia.

- Forrester [4] y Symons [16] presentaron la Metodología propietaria de Forrester Consulting, The Total Economic Impact ${ }^{\mathrm{TM}}$, la cual consiste en la recolección de los beneficios, costos y flexibilidad como los datos de entrada pasando por el filtro de los riesgos para ajustar las cifras y así obtener el ROI (indicador de retorno de inversión).

- También existen las siguientes metodologías: Value Measuring Methodology (Booz Allen Hamilton), Value of Opportunity Methodology (de la firma Gartner), Business Value Index (BVI, desarrollado por Intel), Information Technology Balanced Scorecard (IT BSC, concebida por Robert Kaplan y David Norton), Rapid Economic Justification (REJ, de la firma Microsoft), entre otras. 
De estas metodologías, sólo la metodología MAIGTI habría sido aplicada a la realidad peruana y el procedimiento 059 detalló con mayor precisión los aspectos que se deseaban evaluar. A continuación se explicarán algunos estudios relacionados a la investigación:

- Alfaro [1] realizó un análisis financiero a un caso hipotético de un proyecto de implantación de un sistema de información ERP "World Class" bajo los escenarios optimista, promedio, y pesimista, para una industria de producción de bienes de consumo humano masivo. El resultado para el escenario optimista fue la generación US\$ 4 millones aproximadamente para un período de cuatro años, para el escenario promedio fue US\$ 660.000 en diez años, y para el escenario pesimista se obtuvieron pérdidas de aproximadamente US\$ 6 millones en el mismo período.

- North [14], en el reporte realizado por la entidad Forrester: "El impacto económico total para Microsoft Productos y Servicios", realizó una proyección del impacto económico mediante la metodología Total Economic Impact ${ }^{\mathrm{TM}}$ y retorno de inversión, obteniendo un ROI de 563\% con ajuste del riesgo y un valor actual neto, con ajuste de riesgo de más de US\$33.500.000.

- Hubbard [6], de la Hubbard Decision Research, realizó un informe de proyección acerca del análisis de la relación riesgo/retorno del proyecto “Agilización de reemplazo de PC's de escritorio de la agencia de protección ambiental", siendo el método empleado Applied Information Economics, y se obtuvo un valor presente neto de US\$ 53.800.000 y retorno de la inversión de 4 a 7 años.

La mayoría de los trabajos expuestos estuvieron relacionados a proyectos de aplicaciones de proveedores aún no ejecutados. Las proyecciones, en su mayoría con alto retorno, no fueron corroboradas. Las organizaciones en general desarrollaron una mayor inquietud por adquisiciones de aplicaciones de proveedores; pero no de aplicaciones desarrolladas con sus propios recursos (in-house). A la fecha de la elaboración de la presente investigación no se encontraron investigaciones similares, realizadas en el Perú o en otros países.

\section{OBJETIVO DE LA INVESTIGACIÓN}

El objetivo general fue evaluar el retorno financiero de un proyecto de desarrollo de un sistema de información de carga in-house implantado en una empresa de transporte interprovincial de Lima mediante la variación del procedimiento 059 de la metodología MAIGTI. El objetivo específico fue hallar el valor generado por los módulos del proceso de venta-carga del proyecto de desarrollo de software in-house en la entidad empresarial conocida como "TransProv" y los factores que lo causaron.

\section{DISEÑO METODOLÓGICO DE LA INVESTIGACIÓN}

El proceso de la recolección de datos se realizó de la siguiente manera:

- Paso 1: Definición de las características del proyecto de desarrollo de un sistema de información.

- Paso 2: Definición de la metodología.

- Paso 3: Variación de la metodología para satisfacer las características específicas.

\section{Paso 1: Definición de las características del proyecto de desarrollo de un sistema de información}

Según el Project Management Institute [15], las características de un proyecto son las siguientes:

A. Temporal (cada proyecto tiene un comienzo $y$ un final definido): proyecto de reemplazo culminado y varios subproyectos de mejora culminados y en marcha.

B. Producto, servicio o resultado único (un proyecto crea un entregable único): servicio a varios procesos bajo propios recursos.

C. Elaboración gradual (desarrollar en pasos e ir aumentando mediante incrementos): incremental y modular sin reutilización de inversiones económicas preliminares.

El desarrollo del sistema de información de encomiendas tuvo las características indicadas por el Project Management Institute para un proyecto: temporal, resultado único y elaboración gradual, y aunque haya sido desarrollado por personal a 
tiempo completo de la empresa de transportes, debe considerarse como un proyecto. A continuación se detalla el tipo de inversión y el tipo de disciplina del proyecto:

A. Tipo de inversión: siguiendo la estructura proporcionada por Apfel y Smith [3] se podría clasificar como un tipo de inversión de solución de negocio y, dentro de este tipo, como inversión en mejora de procesos, dado que permite dar soporte a procesos de negocio con la finalidad de solucionar los problemas derivados de la falta de automatización de las ventas de encomiendas.

B. Tipo de disciplina en la ingeniería de Software: según la IEEE [8], este proyecto debería corresponder a la disciplina "Management" del área "Operations Management" debido a la calidad de apoyo a las operaciones del negocio del sistema de información desarrollado.

\section{Paso 2: Definición de la metodología}

Se seleccionaron las metodologías empleadas para evaluar el retorno financiero o el valor de proyectos de desarrollo de sistemas de información en algún estudio previo, obteniéndose las siguientes:

a. Applied Information Economics Methodology

b. Total Economic Impact Methodology

c. MAIGTI Procedimiento 059

d. Total Value of Opportunity Methodology

e. Value Measuring Methodology.

De las metodologías expuestas, se eligió el Procedimiento 059 de la metodología MAIGTI, por las siguientes razones:

a. Implica una mayor adecuación a las características del proyecto de sistema de información en comparación con las demás metodologías, debido a que no está en función de un valor futuro (variables como el riesgo o probabilidad) para determinar el valor generado por el proyecto (ver Tabla 1).

b. Usa el margen de contribución adicional que ha generado la entidad para evaluar la rentabilidad del sistema, por lo que no se necesita cuantificar más variables.

c. Está orientado a las ganancias sobre los ingresos, el cual se relaciona directamente a los módulos de venta-carga del sistema. d. Los indicadores están encaminados a un estado actual (conceptos de Costeo Directo, Free Cash Flow, y Valor Actual Neto), en base a información histórica (no proyecciones).

e. Ha sido aplicado a una realidad nacional.

En la Tabla 1 se observa una tabla de puntuaciones mediante la cual se determinó que el procedimiento 059 de la metodología MAIGTI era el más adecuado, luego de compararlo con las demás metodologías existentes.

El Procedimiento 059 de la metodología MAIGTI se adecuó para satisfacer las siguientes características:

- Establece pautas para los proyectos de implantación modular, propios en un desarrollo de software.

- Establece pautas para los proyectos de implantación incremental (mejoras en módulos ya implantados), propios en un desarrollo de software.

\section{Paso 3: Variación de la metodología para satisfacer las características específicas}

En base a lo expuesto anteriormente se adaptó el procedimiento 059 de la metodología MAIGTI de la siguiente forma:

1. Identificación del proceso de información: módulos asociados al sistema.

2. Identificación del incremento implantado: dado en los procesos del sistema de información así como la variabilidad en los recursos empleados para el desarrollo de la misma.

3. Definición de los períodos de evaluación del proyecto: tiempo de vida de la tecnología de información del proyecto.

4. Definición de la tasa mínima atractiva de retorno de la inversión: debe ser determinada por el directorio.

5. Identificación de los ingresos adicionales: incremento del margen de contribución y los ahorros generados.

6. Identificación de los egresos adicionales: inversiones adicionales y gastos adicionales como resultado de la implantación del proyecto.

7. Cálculo del flujo neto.

8. Cálculo de la generación de valor del proyecto.

9. Verificar la conveniencia del proyecto. 


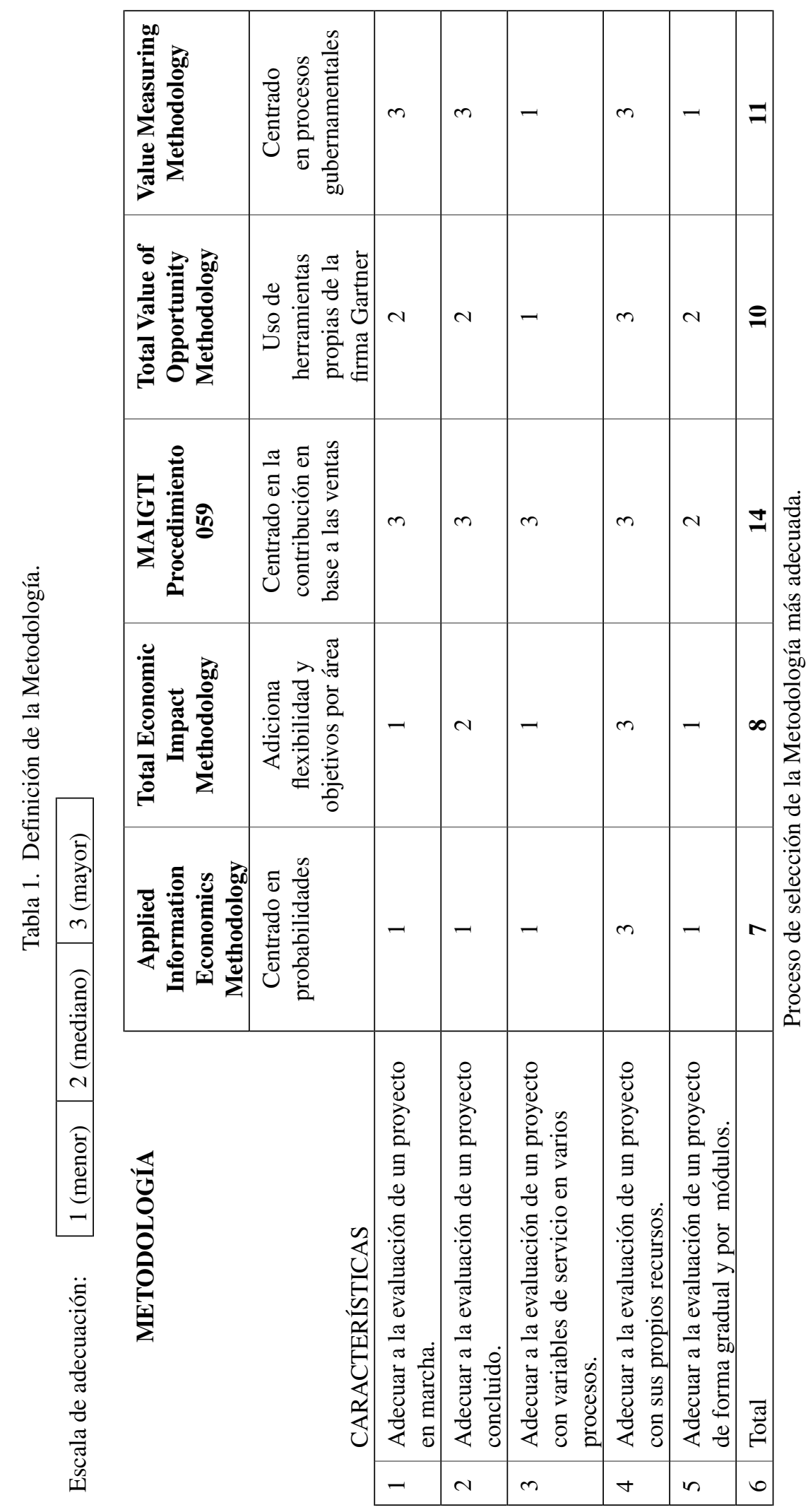




\section{RECOLECCIÓN DE DATOS}

La recolección de datos siguió los nueve pasos descritos en los párrafos anteriores, los cuales están detallados en las siguientes secciones:

Identificación del proceso y módulos asociados al proyecto de sistema de información

A. Definición del Proceso. Se evaluó el "proceso de gestión de venta-carga", debido a:

- La magnitud del proyecto.

- Los módulos de venta tenían casi tres años luego de implantados, tiempo considerable para observar la evolución financiera través del tiempo.

- El proceso de gestión de ventas es crítico y relevante en toda organización.

- Es común que el área de ventas sea la primera en ser afectada en la implantación de un sistema de información.

B. Definición de los módulos asociados. Para ello se identificaron los procedimientos asociados al proceso de venta-carga, y a su vez se separaron las tareas asociadas al sistema de información de las tareas de los procedimientos manuales (ver Tabla 2).

\section{Identificación del incremento modular en el sistema de información}

Para saber cuál ha sido el incremento de los módulos implantados y su porcentaje de dedicación se realizaron entrevistas al personal responsable (jefe y personal antiguo del área de Sistemas y el gerente de carga), obteniéndose las fechas de implantación (corroborando con los registros más antiguos del sistema) y tiempo de dedicación en desarrollo, mantenimiento, soporte, documentación, mejoras, y capacitación (corroborado con los cronogramas de comité, ver Tabla 3).

\section{Definición de los períodos de evaluación del proyecto}

El proyecto se desarrolló desde diciembre 2005 hasta agosto del 2006, mes en el cual dejó de funcionar el anterior sistema de información. El período de evaluación del proyecto fue desde septiembre 2006 hasta agosto 2009.

\section{Definición de la tasa mínima atractiva de retorno} La Gerencia de Carga indicó que la TMAR (Tasa mínima atractiva de retorno) fue $25 \%$, por ser una tasa superior a una tasa libre de riesgo y por ser el rendimiento mínimo que esperaban para sus proyectos. No fue proporcionada mayor información acerca de los detalles del cálculo de la TMAR de la empresa "TransProv".

\section{Identificación de los ingresos adicionales}

$\mathrm{El}$ análisis de los ingresos adicionales incluyó: el cálculo del margen de contribución por producto relacionado al sistema de venta-carga (ver Tabla 4), los resultados de las encuestas realizadas, las entrevistas, y las estadísticas correspondientes al progreso del mercado en carga a nivel nacional.

\section{Identificación de los egresos adicionales}

Los egresos adicionales que se identificaron fueron los siguientes:

A. Costos de Hardware y Software. En total fueron S/. 127.614 nuevos soles. Estos costos fueron divididos en dos grupos:

a) El primero incluye los costos de hardware y software adicional para el desarrollo y mantenimiento de la aplicación, obteniéndose para hardware (servidores, computadores) un total de S/. 3.114 nuevos soles (S/. es el símbolo de nuevos soles, la moneda peruana, vigente durante el período de la investigación) y para licencias de software S/. 45.000 nuevos soles.

b) El segundo incluye los costos de hardware y software adicional para la operatividad del proceso de ventas a nivel de cliente obteniéndose para hardware (computadores, impresoras, concentradores, entre otros equipos) un total de $S / .79 .500$ y para software S/. 0.

B. Costos de recursos humanos. Para hallar estos costos se procedió a colocar el porcentaje estimado de consumo de tiempo por desarrollo de los módulos (ver Tabla 3) multiplicado por el total de nuevos soles invertidos en los honorarios percibidos por los trabajadores, obteniéndose:

a. Antes de implantación (diciembre 2005-septiembre 2006): S/. 70.915.

b. Después de implantación: 


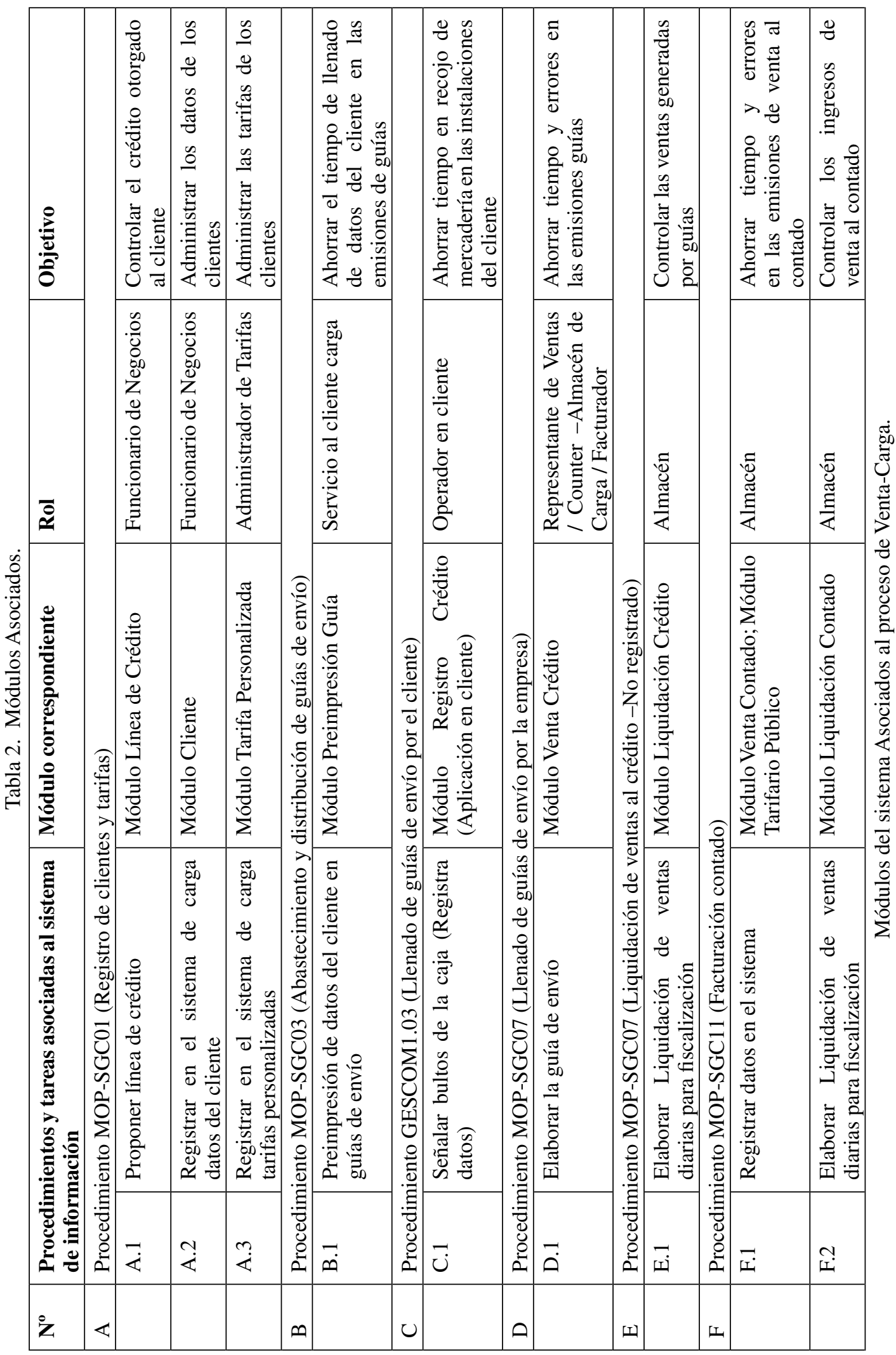




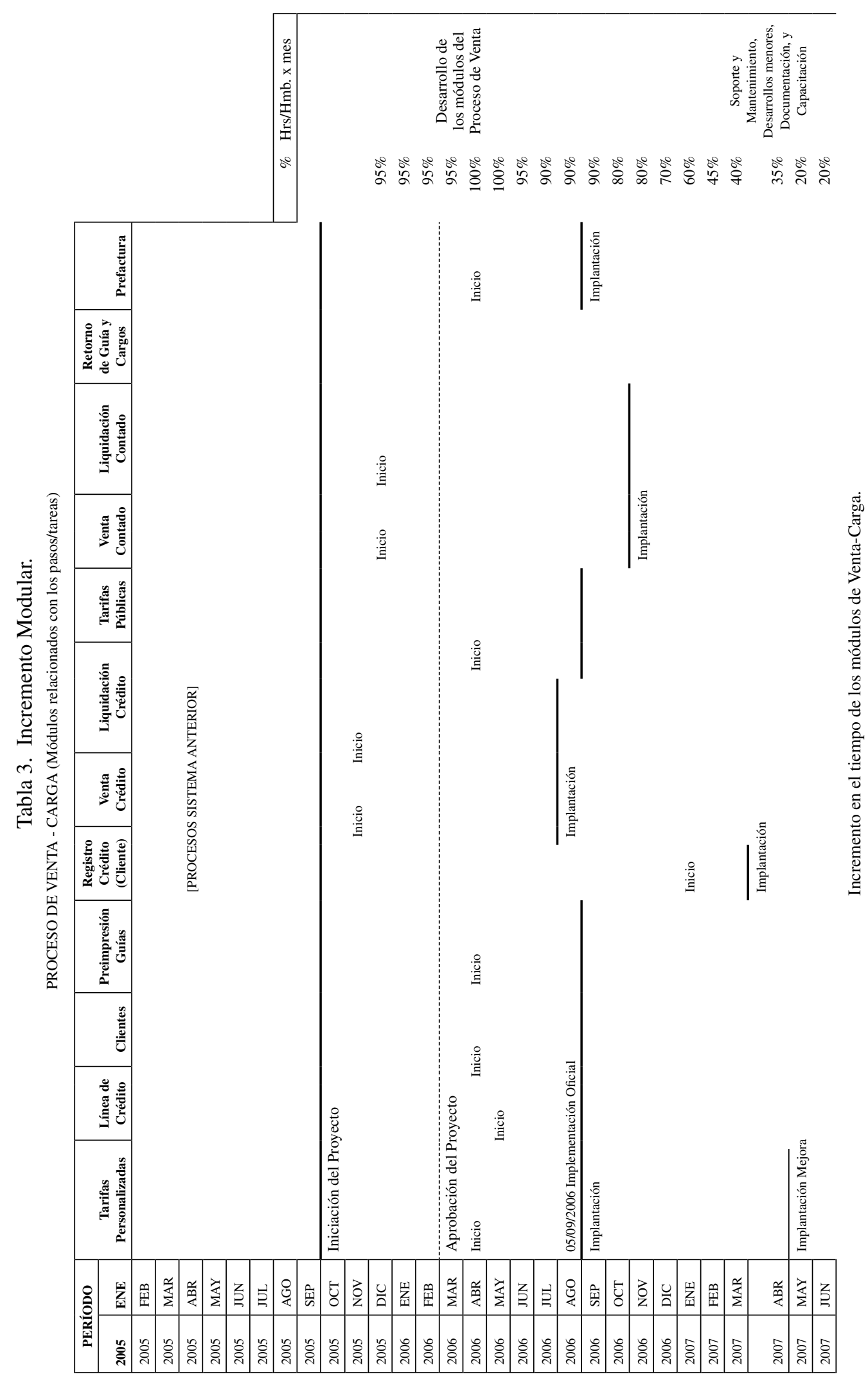


- El Primer año (octubre 2006-septiembre 2007) un total de S/. 46.170.

- Segundo año (octubre 2007-septiembre 2008): S/. 26.425.

- Tercer año (octubre 2008-septiembre 2009): S/. 25.625.

A continuación se detalla la desagregación de valores por producto:

\section{A. Carga Corporativa}

Como se puede observar en la Figura 1, este producto tuvo crecimiento en las ventas durante el año 2007 y el año 2008; sin embargo, en el último trimestre del 2008 tuvo una disminución de ventas en más de S/. 800.000 nuevos soles, debido a una pérdida cuantiosa de mercadería de un cliente, como resultado de la mala gestión por parte de los responsables, viéndose reflejada en los datos de pérdida de mercadería e ingreso de comisiones por parte de los funcionarios.

El proceso de venta con línea de crédito para un cliente corporativo radica en la emisión de guías de envío para luego conglomerarse todo en una sola factura a fin de mes, según sea el caso; por lo tanto, el ingreso de la Carga Corporativa no se da en el punto de venta a través de la emisión del comprobante, sino en el cobro de las facturas que se emiten a los clientes por parte del área de Créditos y Cobranzas. Es por ello que la variabilidad dependió mucho del cobro. Se concluye que el sistema implantado no ejerció una influencia directa con los ingresos del producto Carga Corporativa, debido a que los cambios se debieron a la gestión del cobro y la capacidad del funcionario responsable para aumentar su cartera de clientes.

\section{B. Giros}

La tendencia del producto Giros (envío de dinero del cliente entre oficinas de una empresa de transportes) fue negativa (ver Figura 2). Las bajas y subidas, después de la implementación, estuvieron dentro de la frecuencia correspondiente al período antes de la implementación, ya que el producto fue ingresado en otro sistema y sus datos fueron trasladados mediante procedimientos a nivel de base de datos al sistema de carga, concluyéndose que el producto Giros no estuvo relacionado directamente con los ingresos generados por el sistema de carga.

\section{Encomienda}

En la Figura 3 se puede observar que desde enero 2005 hasta septiembre 2006, las ventas de este producto disminuyeron, iniciando con más de S/. 700.000 y llegando a menos de S/. 450.000 en casi dos años. Luego, este producto presentó un incremento en el trimestre siguiente, coincidentemente con la implantación del sistema de carga, subiendo a más de un millón de nuevos soles en el último trimestre de 2007, en menos de un año después de la implantación. Existió un incremento aproximado de S/. 100.000 para la temporada de fin de año del 2007 y del 2008 (más del 60\% de lo habitual), indicio del aumento de ingresos en temporada navideña. En una entrevista a los contadores de carga, manifestaron que se dio incremento de personal en su área por navidad.

Se dio una disminución de nuevos clientes tanto para los puntos de venta que poseían sistema de información como para los puntos de venta que no poseían un sistema de información. También se dio un aumento de las ventas, con una superioridad inclusive de 10 veces para los puntos de venta que poseían sistema. Por último, el número de comprobantes emitidos indicó una pendiente positiva en aquellas agencias que poseían sistema, y lo contrario sucedió para los demás casos.

El sistema habría ayudado a que los contadores de carga puedan emitir sus comprobantes de manera más rápida, asegurando la lealtad del cliente (mayor uso del servicio por parte de los clientes actuales) del servicio Encomienda, aumentando así las ventas y la estabilidad con respecto a las agencias que no poseían sistema.

A continuación se detalla el resultado de las encuestas realizadas:

A. Tiempo en emisión de comprobantes y cierre de liquidación en puntos de venta

Según se indagó con las encuestas, se obtuvo una reducción de tiempos notable en las emisiones tanto de un comprobante como en las liquidaciones de los contadores de carga en más de $70 \%$.

B. Facturas rechazadas en puntos de venta 


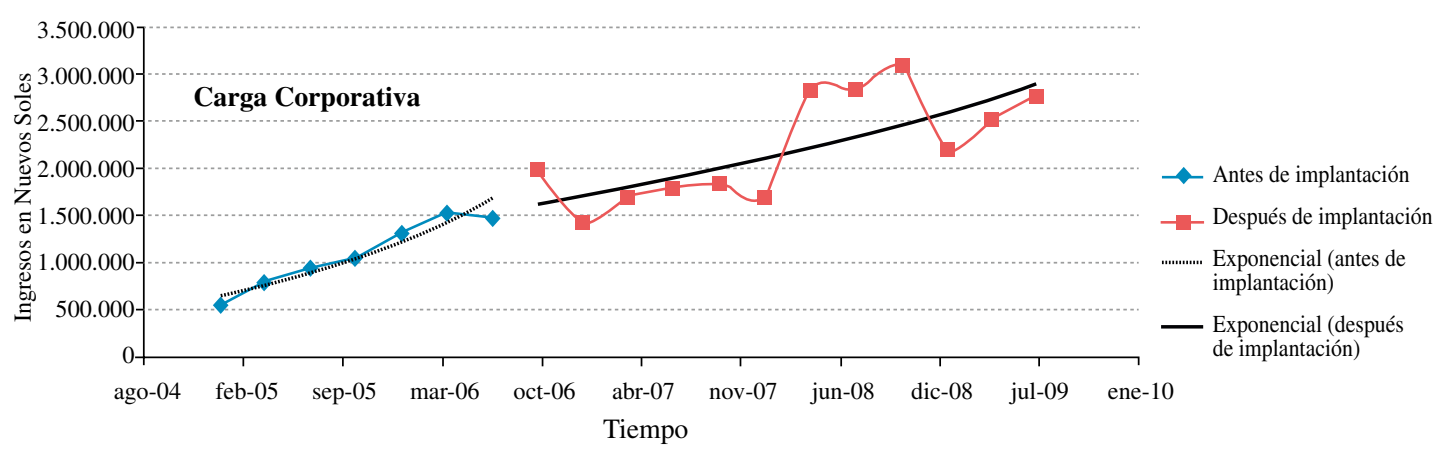

Evolución de las ventas en el producto Carga Corporativa.

Figura 1. Carga Corporativa.

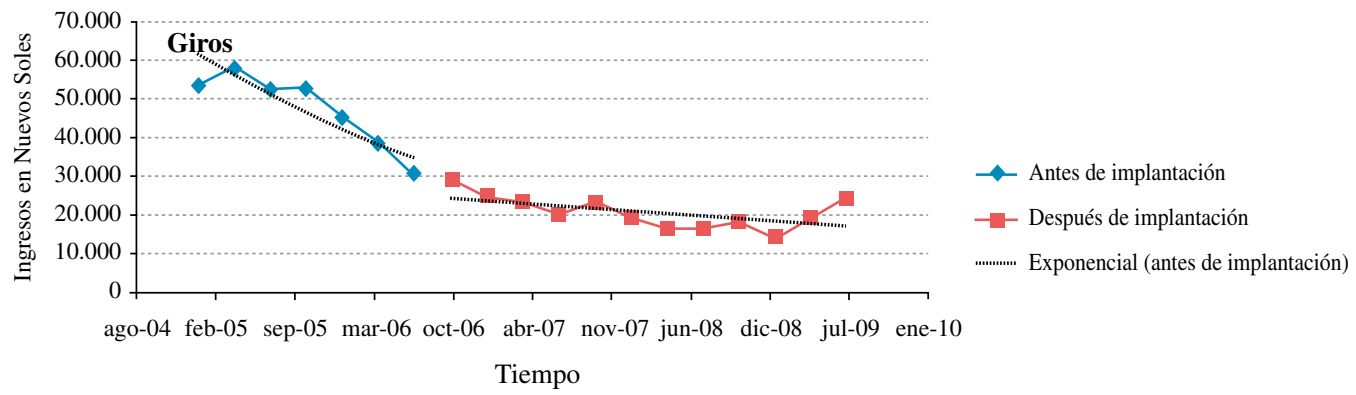

Evolución de las ventas en el producto Giro.

Figura 2. Giros.

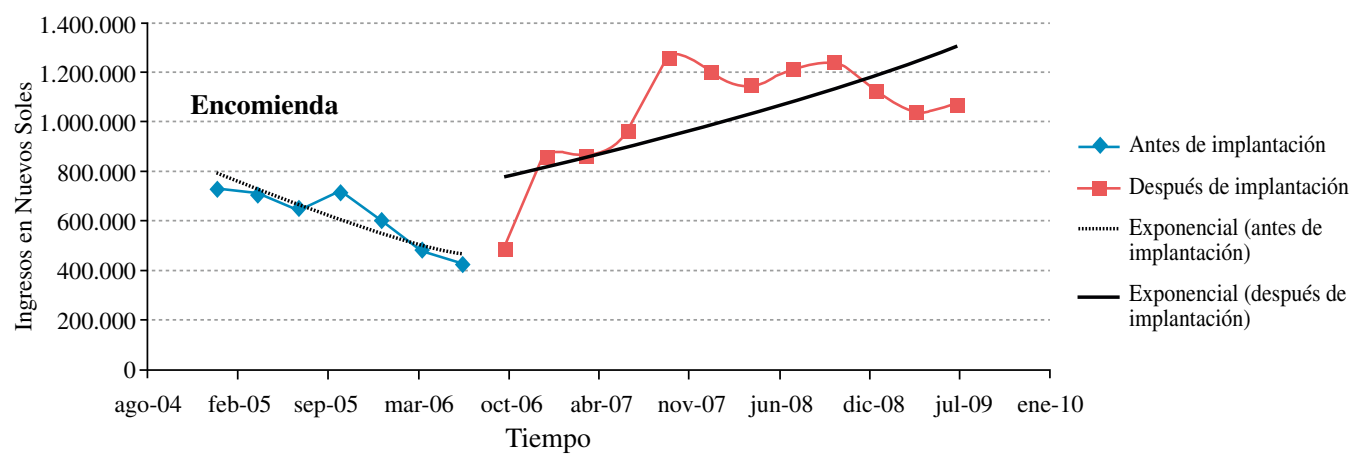

Evolución de las ventas en el producto Encomienda.

Figura 3. Encomienda. 
Mediante las encuestas realizadas se pudo detectar que sin sistema de información existía el rechazo de facturas por parte del cliente (pérdida de venta cuando se emite manualmente) debido a la informalidad que se contemplaba; se obtuvo que antes de la implantación, el número de comprobantes rechazados en promedio era uno al día, y la venta perdida variaba entre S/. 30 y S/. 70 nuevos soles. Si consideramos el número de provincias (19 en total), obtenía un total mensual promedio de S/. 22.800 nuevos soles.

\section{Resultado de las entrevistas realizadas}

a. Cierre y apertura de agencias

Mediante una entrevista se pudo detectar la existencia de cierres masivos de locales en Lima y provincias debido al monto de sus ventas, tanto en Carga como en Pasajes. Para el 2005 se habían cerrado nueve agencias, cinco agencias en el 2006, dos agencias en el 2007 y una agencia en el 2008. En ese mismo año se abrió una agencia y para el 2009 se abrió otra agencia en el norte. El cierre de agencias estuvo relacionado a la disminución de las ventas de los productos de Encomienda.

\section{b. Campañas de Marketing}

Manifestaciones de los responsables del área de Marketing, Sistemas y Gerencia indicaron que no hubo una promoción de marketing en Encomienda durante el último trimestre de 2006, concluyéndose que el crecimiento dado luego de la implantación no fue producto de una promoción de Marketing y que sí es probable que haya ayudado a incrementar las ventas.

D. Crecimiento del mercado de transporte de Carga a nivel nacional

a. Empresas de transporte de carga autorizadas Los datos estadísticos de las variaciones en ventas de las empresas de transporte autorizadas, proporcionados por la Dirección General de Transporte Terrestre del Ministerio de Transportes y Telecomunicaciones [12], mostraron un crecimiento constante en el período correspondiente del 2000 al 2006, a razón de $1 \%$ anual tanto a nivel nacional como en Lima (con más del $75 \%$ sobre el total).

b. Parque vehicular de empresas de transporte de carga nacional autorizadas
Los datos estadísticos del parque vehicular de las empresas de transporte de carga nacional autorizadas, proporcionados por la Dirección General de Transporte Terrestre del Ministerio de Transportes y Telecomunicaciones [13], al igual que el caso anterior, indicaron un incremento constante en el período del 2000 al 2006, a razón de $3 \%$ anual a nivel nacional y $2 \%$ en Lima.

Para los dos casos presentados existieron crecimientos constantes en cuanto a transporte de carga a nivel nacional (períodos del 2000 al 2006), lo que implicaba un crecimiento positivo en todos los productos de Carga, no correspondiendo así con los datos de la empresa "TransProv" la cual ha tenido disminuciones constantes en las ventas de sus productos Giros y Encomienda, antes de la implantación en el período del 2005 al 2006.

Finalmente, para este análisis de los ingresos se puede afirmar la relación directa entre el crecimiento del producto Encomienda con la implantación del sistema de carga (módulos de venta-carga).

\section{Cálculo del Flujo Neto}

La ventas del producto Encomienda fueron afectadas positivamente por el sistema de carga (módulo de venta - carga). Se realizó el cálculo de valor sobre la base de los ingresos del producto Encomienda. Para el flujo neto se sumaron los ingresos adicionales (margen de contribución adicional y ahorros) y se restaron los egresos adicionales (inversiones antes de implantación más gastos del proyecto). El cálculo del margen de contribución adicional se dividió en dos partes: a) cálculo del margen de contribución del producto Encomienda para los años después de la implantación y el margen antes de la implantación (ver Tabla 5), b) cálculo del margen de contribución adicional con respecto al año antes de la implantación en el período de octubre 2005 a septiembre 2006 (año 0 , ver Tabla 6); obteniéndose a) - S/. 70.915 para el año 0, b) S/. 261.231 para el año 1, c) S/. 832.281 para el año 2 y d) S/. 992.708 para el año 3.

\section{Cálculo de la generación de valor del proyecto}

Para el cálculo del valor neto actual, se aplicó la Tasa Mínima Atractiva de Retorno de 25\% al final del primer año, al final del segundo año y al final del tercer año después de la implantación (ver Tabla 7), obteniéndose para el primer año un valor neto 
Tabla 4. Porcentaje de los productos sobre las ventas

\begin{tabular}{|c|c|c|c|c|c|c|c|c|}
\hline & AÑO 0 & Porcentaje & AÑO 1 & Porcentaje & AÑO 2 & Porcentaje & AÑO 3 & Porcentaje \\
\hline Encomienda & S/. 2.238.699 & $29 \%$ & S/. 3.174 .826 & $31 \%$ & S/. 4.834.310 & $34 \%$ & S/. 4.490 .903 & $29 \%$ \\
\hline Carga Corporativa & S/. 5.377.825 & $69 \%$ & S/. 6.902 .414 & $68 \%$ & S/. 9.216.478 & $65 \%$ & S/. 10.581.136 & $69 \%$ \\
\hline Giros & S/. 166.292 & $2 \%$ & S/. 96.311 & $1 \%$ & S/. 74.120 & $1 \%$ & S/. 74.823 & $2 \%$ \\
\hline Total Ingresos & S/. 7.782.815 & $100 \%$ & S/. 10.173.550 & $100 \%$ & S/.14.124.908 & $100 \%$ & S/. 15.337.175 & $100 \%$ \\
\hline
\end{tabular}

Porcentaje de los productos sobre las ventas.

Tabla 5. Margen de contribución del producto de encomienda.

\begin{tabular}{|l|r|r|r|r|}
\hline & \multicolumn{1}{|c|}{ AÑO 0 } & \multicolumn{1}{c|}{ AÑO 1 } & \multicolumn{1}{c|}{ AÑO 2 } & \multicolumn{1}{c|}{ AÑO 3 } \\
\hline Total Ingresos Encomienda & S/.2.238.699 & S/.3.174.826 & S/.4.834.310 & S/.4.490.903 \\
\hline Total Gastos Variables & S/. 1.222.144 & S/.3.141.225 & S/.6.102.046 & S/. 5.414.460 \\
\hline Porcentaje Encomienda sobre venta & $29 \%$ & $31 \%$ & $34 \%$ & $29 \%$ \\
\hline $\begin{array}{l}\text { Total Gastos * Porcentaje sobre venta } \\
\text { de Encomienda }\end{array}$ & S/.351.545 & S/.980.272 & S/.2.088.451 & S/. 1.585.417 \\
\hline Total Margen de contribución & S/.1.887.153 & S/.2.194.555 & S/.2.745.859 & S/.2.905.486 \\
\hline
\end{tabular}

Cálculo para hallar el Margen de contribución del producto Encomienda.

Tabla 6. Diferencia con respecto al año 0.

\begin{tabular}{|l|r|r|r|}
\cline { 2 - 4 } \multicolumn{1}{c|}{} & AÑO 1 Adicional & AÑO 2 Adicional & AÑO 3 Adicional \\
\hline Diferencia con respecto al año 0 & S/.307.401 & S/.858.706 & S/. 1.018.333 \\
\hline
\end{tabular}

Cálculo de la diferencia del margen de contribución con respecto al año 0.

Tabla 7. Cálculo del Valor.

\begin{tabular}{|l|l|l|l|}
\hline Año 0 & Año 1 & Año 2 & Año 3 \\
\hline
\end{tabular}

Ingresos adicionales

\begin{tabular}{|l|r|r|r|r|}
\hline \multicolumn{2}{|l|}{ Margen de contribución adicional } & S/. 307.401 & S/. 858.706 & S/. 1.018.333 \\
\hline Ahorros & S/.0 & S/.0 & S/.0 & S/.0 \\
\hline Total & S/. 0 & S/. 307.401 & S/. 858.706 & S/. 1.018.333 \\
\hline
\end{tabular}

Egresos adicionales

\begin{tabular}{|l|r|r|r|r|}
\hline Inversiones & S/. 70.915 & & & \\
\hline Gastos & & S/. 46.170 & S/. 26.425 & S/. 25.625 \\
\hline Total & S/. 70.915 & S/. 46.170 & S/. 26.425 & S/. 25.625 \\
\hline
\end{tabular}

Flujo Neto

TMAR

VNA 1 año

VNA 2 años

VNA 3 años

\begin{tabular}{|c|c|c|c|}
\hline S/. (70.915) & S/. 261.231 & S/. 832.281 & S/. 992.708 \\
\hline $25 \%$ & & & \\
\hline S/. 138.070 & & & \\
\hline S/. 670.730 & & & \\
\hline S/. 1.178 .996 & & & \\
\hline
\end{tabular}

Cálculo del valor para el producto Encomienda. 
actual positivo de S/. 138.070 (aproximadamente US\$ 48.446), casi quintuplicándolo para el segundo año con S/. 670.730 (aproximadamente US\$ 235.344), y llegando para el tercer año a S/. 1.178.996 nuevos soles (aproximadamente US\$ 413.683).

\section{Verificar la conveniencia del proyecto}

El nuevo sistema de información de encomiendas permitió la generación de valor de la empresa "TransProv" por S/. 1.178.996 (aproximadamente US\$ 413.683) en un período de tres años y seguirá generando valor, por lo cual se concluye que el proyecto fue conveniente para la empresa.

\section{CONCLUSIONES}

A continuación se detallan las conclusiones de la investigación:

A. La adecuación del procedimiento 059 de la metodología MAIGTI evaluó el retorno financiero de un proyecto de desarrollo de sistema de información de carga in-house en empresa de transporte interprovincial de Lima.

B. La variación del procedimiento 059 de la metodología MAIGTI fue congruente con las características correspondientes a los módulos de venta del proyecto de desarrollo de sistema de información in-house implantado para su evaluación.

C. Los módulos correspondientes al proceso de venta-carga en el sistema de información inhouse implantado son rentables a partir del primer año con S/. 138.070, generando para el tercer año el valor de S/. 1.178.996.

D. El producto "Carga corporativa" fue afectado por las operaciones realizadas por los funcionarios de negocio y por el área de Créditos y cobranzas.

E. El producto "Giros" fue ingresado en un sistema de información exógeno al evaluado.

F. El producto "Encomienda" estuvo directamente relacionado con el punto de venta; así lo demuestran sus datos estadísticos.

G. Los ingresos del producto "Encomienda" en el período de la implantación no variaron por ninguna de las siguientes razones:
- Campañas de Marketing durante el período de implantación.

- Apertura masiva de agencias en Lima y provincias.

- Correlación entre el crecimiento general del transporte de carga en los últimos nueve años con los ingresos por ventas del producto Encomienda.

H. Los ingresos del producto "Encomienda" después de la implantación variaron por el aumento de la lealtad del cliente (mayor frecuencia en el envío de mercadería), gracias a los siguientes factores:

- Reducción de 70\% del tiempo en la emisión de comprobantes gracias a la automatización del proceso.

- Se logró evitar pérdidas de ventas de facturas emitidas manualmente que finalmente no se concretaban, con un valor mensual aproximado de S/. 23.000 mensuales.

En resumen, mediante la adecuación del procedimiento 059 de la metodología MAIGTI se ha podido evaluar si los módulos correspondientes al proceso de ventacarga del sistema de información in-house implantado de la empresa "TransProv", afectaron positivamente al producto "Encomienda", concluyéndose que el sistema desarrollado permitió incrementar las ventas gracias al aumento de la lealtad del cliente fruto de una atención más rápida y la satisfacción en las emisiones de facturas impresas.

\section{RECOMENDACIONES PARA INVESTIGACIONES FUTURAS}

A continuación se detallan las recomendaciones para investigaciones futuras:

A. Realizar un estudio para saber cuántas empresas de Lima metropolitana, en la actualidad, han desarrollado estimaciones de inversión antes de desarrollar sus proyectos de sistema de información y cuántas de ellas lo realizan después.

B. Ampliar el número de empresas bajo análisis, con la finalidad de evaluar la influencia del crecimiento del mercado junto a la influencia de la implantación del software de venta-carga. 
C. Aplicar la solución planteada a una muestra representativa del sector de transporte de carga así como a otros sectores.

D. Analizar de qué manera se aplicaría el procedimiento 059 de la metodología MAIGTI en entidades sin fines de lucro.

E. Calcular el valor de retorno de un caso con más de una metodología para comparar los resultados y obtener el más adecuado para la tecnología de información aplicada.

F. Evaluar las relaciones entre los resultados del retorno de inversión y el tipo de inversión de tecnología de información, comparándolos con las propuestas de Lucas [10].

G. Comparar el retorno de inversión de las tecnologías de información implantadas bajo un marco de trabajo como ITIL, COBIT, VALIT, entre otros, con el retorno de inversión de tecnologías de información que no hayan sido implantadas con marcos de trabajo.

H. Realizar una investigación que permita saber por qué las organizaciones que brindan soluciones de tecnología de información no rinden cuentas del retorno de inversión de sus productos (bienes o servicios) a las organizaciones que las contrataron.

I. Realizar comparaciones entre el valor de retorno de los proyectos de desarrollo de sistemas de información desarrollados por la propia entidad (in-house) y los desarrollados por terceros (Outsourcing).

\section{AGRADECIMIENTOS}

Agradecemos a la Universidad Privada Norbert Wiener por las facilidades brindadas para el desarrollo de la presente investigación.

\section{REFERENCIAS}

[1] E.A. Alfaro. "Los ERPs ¿Generan o Destruyen Valor?". IV Congreso Internacional de Ingeniería de Sistemas. Trujillo, Perú. 22 de Junio de 2007.
[2] E.A. Alfaro. "MAIGTI: Metodología para la Auditoría Integral de la Gestión de las Tecnologías de información". CISAISI XII - Congreso Internacional Sudamericano de Ingeniería de Sistemas, Computación e Informática. Arequipa, Perú. 7 de octubre de 2008.

[3] A. Apfel and M. Smith. "TVO Methodology: Valuing IT Investments via the Gartner Business Performance Framework", p. 16. Date of publication: March 3, 2008. Date of visit: November 3, 2009. URL: http://www. cidge.gob.mx/doc/TVO_Gartner_BPF.pdf

[4] Forrester Research Inc. "The Total Economic Impact ${ }^{\mathrm{TM}}$ of Using ThoughtWorks' Agile Development Approach", p. 18. Date of publication: June 1, 2008. Date of visit: November 3, 2009. URL: http:// www.thoughtworks.com/sites/www. thoughtworks.com/files/files/TEI-media.pdf.

[5] J. Gantin. "Assessment of IT Value Delivery at a Large Nordic Bank", p. 105. Master Thesis of KTH School of Electrical Engineering. KTH Publications. Stockholm, Sweden. 2006.

[6] D. Hubbard. "Applied Information Economics (AIE) Analysis of The Desktop Replacement Policy for the Environmental Protection Policy", p. 35. Date of publication: August, 2003. Date of visit: November 3, 2009. URL: http://www.federalelectronicschallenge.net/ resources/docs/aie_desktop.pdf.

[7] N. Huber. "Gartner: Firms Waste $£ 351$ bn Each Year on Ill-conceived IT Projects?", p. 1. Date of publication: March 21, 2002. Date of visit: November 3, 2009. URL: http://www.computerweekly. com/Articles/2002/03/21/185891/ Gartner-firms-waste-163351bn-each-yearon-ill-conceived-IT.htm.

[8] IEEE. "Guide to the Software Engineering Body of Knowledge". The Institute of Electrical and Electronics Engineers. Los Alamitos, California, USA., p. 204. 2004. ISBN 0-7695-2330-7.

[9] IT Governance Institute. "IT Governance Global Status Report 2008”. IT Governance Institute, p. 73. Rolling Meadows, Illinois, USA. 2008. ISBN: 978-1-60420-064-5. 
[10] H. Lucas. "La tecnología de la información y la paradoja de la productividad". Oxford University Press Inc. Vol. 1, p. 261. New York, USA. 2000. ISBN: 978-9-70613-513-1.

[11] A. McCue. "Most Companies "Guess" Tech ROI". Date of publication: May 24, 2009. Date of visit: November 3, 2009. URL: http://www.businessweek.com/globalbiz/ content/may2006/gb20060524_374324. htm.

[12] Ministerio de Transportes y Telecomunicaciones. "Empresas de transporte de carga nacional autorizadas, según región: 2000-2008". 2009. Fecha de consulta: 03 de noviembre de 2009. URL: http://www. mtc.gob.pe/estadisticas/index.html.

[13] Ministerio de Transportes y Telecomunicaciones. "Parque vehicular de empresas de transporte de carga nacional autorizadas, según antigüedad: 2000-2008”. 2009. Fecha de consulta: 03 de noviembre de 2009. URL: http://www.mtc.gob.pe/estadisticas/index. html.

[14] J. North. "The Total Economic Impact" Of Microsoft Unified Communications Products and Services". Forrester Research, Inc. Cambridge, Massachusetts, USA., p. 47. 2007.

[15] Project Management Institute. "Guía de los Fundamentos de la Dirección de Proyectos (Guía del PMBOK®)". PMI Publications. Pennsylvania, USA., p. 407. 2004. ISBN: 1-930699-73-5.

[16] C. Symons. "Measuring The Business Value Of IT". Forrester Research, Inc. Cambridge, Massachusetts, USA. 2006. 